\title{
Competências Digitais Docentes e o Processo de Ensino Remoto Durante a Pandemia de Covid-19
}

\section{Digital Teaching Competence and the Remote Teaching Process during the Covid-19 Pandemic}

Ismael Jung Sanchotene ${ }^{1 *}$

Phillip Vilanova Ilha'

Raquel Ruppenthal ${ }^{1}$

Patrícia Becker Engers ${ }^{1}$

1Universidade Federal do PampaUNIPAMPA Campus Uruguaiana.

Endereço: BR 472 - Km 585 - Caixa Postal 118 - Uruguaiana - RS. Brasil

*ismaeljungsanchotene@hotmail.com

\section{Resumo}

O objetivo do presente estudo foi identificar as competências digitais dos professores da rede municipal de ensino e a influência das tecnologias digitais de comunicação e informação durante o ensino remoto na pandemia de Covid-19. Trata-se de um estudo caracterizado como descritivo-exploratório. Participaram deste um total de 121 docentes de todas as áreas do conhecimento (linguagens, matemática, ciências humanas, ciências da natureza e ensino religioso). Para a recolha dos dados foi aplicado questionário baseado no Quadro Europeu de Competências Digitais para professores, além de questões sobre o desenvolvimento das aulas durante a pandemia. Dentre os participantes, $75,2 \%$ eram do sexo feminino. Quanto à formação inicial dos professores, a prevalência foi de $34,7 \%$ na área de linguagens; 54\% não possuíam pós-graduação. Em relação ao desenvolvimento das aulas durante o isolamento social, destacou-se, entre os recursos utilizados, o material impresso como principal ferramenta pedagógica (84,3\%). O nível de competência digital predominante entre os professores era o B1-Integradores $(40,5 \%)$, indicando que eles experimentam tecnologias digitais em diferentes contextos e utilizam-nas em suas aulas. Em relação à área na qual o professor apresentava mais competência, observou-se média superior nas áreas de envolvimento profissional e capacitação dos estudantes (1,9 pontos). Concluiu-se que os professores estudados tinham mais proficiência digital para utilização profissional, entretanto com carências no que tange à utilização das tecnologias digitais na educação visando potencializar e apoiar estratégias pedagógicas centradas no desenvolvimento ativo do estudante.

Palavras-chave: Competência digital. Ensino remoto. Educação básica.

\section{COMO CITAR ESTE ARTIGO}

ABNT: SANCHOTENE, I. J. et al. Competências Digitais Docentes e o Processo de Ensino Remoto Durante a Pandemia de Covid-19. EaD em Foco, v. 10, n. 3, e1303, 2020.

DOI: https://doi.org/10.18264/eadf.v10i3.1303 


\section{Abstract}

The aim of the study was to identify the digital skills of teachers in the municipal education network and the influence of digital communication and information technologies during remote education in the Covid-19 pandemic. Regarding the methodological processes, this is a study with a qualitative and quantitative approach. A total of 121 professors from all areas of knowledge participated. To collect data, a questionnaire based on the European Digital Skills Framework for teachers was applied, in addition to questions about the development of classes during the pandemic. Among the main results obtained, most of the female gender stands out, 54\% do not have a postgraduate degree and the prevalence of language areas. Regarding the resources used, we highlight the use of printed material as the main pedagogical tool and the evolution of a newcomer to an integrator. The results show that during the teaching process most students are not learning, and that the level of competence indicated that teachers have experimented and used digital technologies in different contexts in their classes. However, we highlight the highest digital proficiency for professional use, and the lowest proficiency in using digital technologies in education in order to enhance and support pedagogical strategies that are central to the student's active development.

Keywords: Digital competence. Remote teaching. Basic education.

\section{Introdução}

O coronavírus é um inimigo invisível que parou o mundo e provocou um número significativo de mortes; por isso, vem sendo considerado como a maior crise sanitária do século XXI. No Brasil, as primeiras ações ligadas à pandemia de Covid-19 começaram em fevereiro de 2020, e o primeiro caso de Síndrome Respiratória Aguda Grave Coronavírus 2 (SARSCoV-2) foi diagnosticado em um homem de 61 anos com histórico de viagem à Itália. Esse vírus é o causador da patologia denominada, pela Organização Mundial da Saúde (OMS), como COVID-19 (BRASIL, 2020).

Desde a chegada ao país, a pandemia fechou muitas escolas, universidades, tratando-se de uma das medidas de contenção da doença e diminuição do contágio. E, nesse contexto, tornou-se um desafio a relação ensino-aprendizagem através do trabalho remoto do professor e estudantes, descrito, por Gomes et al. (2020), como "ensino remoto emergencial", que ocasionou a necessidade de inclusão e o aprendizado rápido de novas tecnologias.

Entretanto, a presença das Tecnologias Digitais da Informação e Comunicação (TDIC) no ensino remoto, em consequência do isolamento social, tornou-se indispensável no nosso dia a dia e tem alterado visivelmente os meios de comunicação e a maneira como nos comunicamos. A realização de tarefas por intermédio das TDIC apresenta características que são importantes para o processo de construção de conhecimento (AUTRAN; BORGES, 2016). A Base Nacional Comum Curricular (BRASIL, 2017, p. 39) traz, em seu texto, que já reconhece o uso na tecnologia em sala de aula, ao afirmar que se deve "utilizar processos e ferramentas educativas, inclusive tecnologias digitais disponíveis, para modelar e resolver problemas cotidianos, sociais e de outras áreas de conhecimento, validando estratégias e resultados".

Contudo é necessário ao professor competências e habilidades para mediar o processo de ensino-aprendizagem a partir das TDIC. Segundo Ferrari (2012), as competências digitais docentes estão rela- 
cionadas a um conjunto de conhecimentos, habilidades, atitudes e estratégias que possibilite a realização de diferentes atividades socialmente valorizadas com uso das TDIC, tais como a resolução de problemas, gerenciamento da informação, colaboração, criação e compartilhamento de conteúdo.

Assim, torna-se importante estudar e analisar as competências digitais dos professores no contexto do ensino remoto da pandemia, pois estes podem utilizar as tecnologias como estratégias de ensino-aprendizagem e de avaliação, criando e compartilhando recursos educacionais que possibilitem estimular o envolvimento profissional do docente (FERRARI, 2012; CHOI; CRISTOL; GIMBERT, 2018).

No atual contexto de isolamento social, como estratégia de combate à pandemia de COVID-19, as TDIC mostraram-se ainda mais relevantes para viabilização do ensino. Todavia, é fundamental considerar as condições de acesso, compreensão e habilidades destas tecnologias tanto no que concerne aos professores como aos estudantes. Segundo Gomes et al. (2020), criar estratégias de ensino remoto são importantes meios de contenção dos efeitos do distanciamento social; no entanto, as evidências sugerem que inúmeras lacunas serão criadas na interação professor/estudante.

Para tal, o educador não precisa ser detentor do conhecimento técnico sobre o uso das ferramentas digitais, mas é necessário que tenha uma compreensão básica para ser mediador do processo de ensino-aprendizagem, proporcionando ao estudante reflexões e competências para melhores usos possíveis das TDIC (CASTAÑEDA; ESTEVE; ADELL, 2018).

Sob tal conjuntura, justifica-se o desenvolvimento desta pesquisa a respeito das competências digitais dos professores e o uso das TDIC na Educação Básica, durante o ensino remoto, na pandemia de COVID-19, visto que as informações sobre as implicações do desenvolvimento das competências digitais dos professores e o impacto no processo educacional poderão fornecer subsídios para auxiliar na sua formação inicial e continuada.

Para tanto, o presente estudo teve como objetivo o de identificar as competências digitais dos professores e a influência das TDIC durante o ensino remoto, na pandemia de Covid-19.

\section{Desenvolvendo o artigo}

\subsection{Metodologia}

Situada na abordagem quali-quantitativa, a presente pesquisa é caracterizada, quanto aos objetivos, como descritiva-exploratória. As pesquisas com objetivos descritivo-exploratórios buscam proporcionar mais finalidade com o problema visando torná-lo mais explícito ou visando construir hipóteses (GIL, 2012).

A população em estudo contemplou professores do ensino fundamental da rede municipal de uma cidade localizada na região sudoeste do estado do Rio Grande do Sul. Para tanto, participaram 121 docentes que estavam em regência de classe e aceitaram participar voluntariamente da pesquisa, das seguintes áreas do conhecimento: linguagens, matemática, ciências da natureza, ciências humanas, ensino religioso e pedagogia. Para a coleta de dados, inicialmente, foi realizado contato com a Secretaria Municipal de Educação, solicitando anuência para a realização da pesquisa. Posteriormente, a pesquisa foi apresentada aos coordenadores pedagógicos, sendo-lhes solicitado que o link de acesso ao instrumento de coleta de dados fosse compartilhado com os professores do ensino fundamental. Para os participantes da pesquisa, ao acessar o instrumento de coleta de dados, foram requeridos previamente a leitura e o aceite do Termo de Consentimento Livre e Esclarecido.

Como instrumento de coleta de dados, foi utilizado um questionário baseado no Quadro Europeu de 
Competências Digitais para Professores, conhecido como DigCompEdu, validado para a língua portuguesa por Dias-Trindade, Moreira e Nunes (2019). O quadro europeu está estruturado em seis áreas de análise: envolvimento profissional; recursos digitais; ensino e aprendizagem; avaliação; capacitação dos estudantes; promoção da competência digital dos estudantes. Cada uma das seis áreas focaliza diferentes aspectos profissionais dos professores; todas, juntas, compreendem um conjunto de 22 perguntas com quatro níveis de resposta cada uma. Foram atribuídos pontos de zero, para o primeiro nível, a quatro pontos, para o último, e a cotação total das 22 perguntas foi de 88 pontos.

Foram inclusas, ainda, ao questionário, questões abertas relacionadas à organização das aulas e percepções dos professores sobre TDIC e ensino remoto. O questionário foi aplicado de forma on-line pelo aplicativo Google Forms; desta forma, os professores puderam responder virtualmente e mantiveram protegido o anonimato.

Para a classificação da competência digital docente, foram utilizadas as caegorias de análise do DigCompEdu (REDECKER; PUNIE, 2017), sendo: A1-Recém-chegado (com até 18 pontos); A2-Exploradores (entre 19 e 32 pontos); B1-Integradores (entre 33 e 47 pontos); B2-Especialistas (entre 48 e 62 pontos); C1-Líderes (entre 63 e 77 pontos); C2-Pioneiros (mais de 77 pontos), conforme é apresentado na Tabela 1.

Tabela 1: Níveis de proficiência e perfis profissionais

\begin{tabular}{|c|c|c|}
\hline Nível & Denominação & Perfil Profissional \\
\hline A1 & Recém-chegado(a) & $\begin{array}{l}\text { Tem oportunidade para começar a melhorar a utilização das tecno- } \\
\text { logias digitais no processo de ensino. }\end{array}$ \\
\hline $\mathrm{A} 2$ & Explorador(a) & $\begin{array}{l}\text { Tem consciência do potencial das tecnologias digitais e tem interes- } \\
\text { se em utilizá-las na melhoria da sua prática pedagógica. }\end{array}$ \\
\hline B1 & Integrador(a) & $\begin{array}{l}\text { Experimenta tecnologias digitais em diferentes contextos e integra- } \\
\text {-as em suas aulas. }\end{array}$ \\
\hline B2 & Especialista & $\begin{array}{l}\text { Usa com confiança e criatividade uma série de tecnologias digitais, } \\
\text { selecionando-as de acordo com o que será ensinado. }\end{array}$ \\
\hline $\mathrm{C} 1$ & Líder & $\begin{array}{l}\text { Tem uma abordagem consistente e abrangente em relação à utili- } \\
\text { zação de tecnologias digitais para melhorar a sua prática pedagógi- } \\
\text { ca, possuindo amplo repertório. Compartilha seus conhecimentos. }\end{array}$ \\
\hline $\mathrm{C} 2$ & Pioneiro & $\begin{array}{l}\text { Questiona a adequação de práticas digitais e pedagógicas contem- } \\
\text { porâneas. Busca inovar constantemente e experimenta tecnologias } \\
\text { inovadoras. }\end{array}$ \\
\hline
\end{tabular}

Fonte: Trindade, Moreira e Nunes (2019, p. 4).

Para análise quantitativa, foi utilizada a estatística descritiva e inferencial através do programa Statistical Package for Social Science for Linux versão 24. Já para a análise qualitativa, das questões abertas, foi empregada a análise de conteúdo proposta por Bardin (2011), mediante a análise categorial.

O estudo foi conduzido de acordo com os princípios éticos, conforme a Resolução n. ${ }^{\circ}$ 510/16, que regulamenta as pesquisas com seres humanos no Brasil.

\section{Resultados e Discussão}

Participaram do estudo 121 professores do Ensino Fundamental de um município da fronteira oeste do Rio Grande do Sul que estavam em regência de classe. Desses, 75,2\% eram do gênero feminino e 24,8\%, do gênero masculino, com média de idade de 42,7 anos $( \pm 9,3)$ e com idade mínima e máxima de 23 e 65 anos, respectivamente. 
Em relação à média de tempo de formação acadêmica, esta foi de 18,1 anos $( \pm 8,3)$, com uma representação de sujeitos em todas as áreas do conhecimento, com maior prevalência na área das linguagens $(34,7 \%)$, seguido da pedagogia (16,5\%), matemática (11,6\%), ciências humanas $(11,6 \%)$, ciências da natureza $(10,7 \%)$, mais de uma área de formação $(9,9 \%)$ e ensino religioso $(5 \%)$. A maior concentração dos sujeitos na área das linguagens é justificada pela abrangência da própria área, pois, esta, integra número superior de componentes quando comparado com as demais áreas, sendo: Língua Portuguesa, Arte, Educação Física e Língua inglesa ou espanhola. Ainda quanto à formação, destaca-se que 54,6\% dos professores não possuíam pós-graduação; já 41,3\% tinham especialização e 4,1\%, mestrado.

Após analisar o perfil acadêmico e profissional dos professores participantes do estudo, procurou-se constatar o desenvolvimento das aulas durante o isolamento social. Para tanto, na Tabela 2, constam as informações pertinentes ao desenvolvimento das aulas, aos recursos didáticos utilizados e à percepção dos professores referente à aprendizagem dos estudantes.

Tabela 2: Desenvolvimento das aulas durante o isolamento social e a percepção de aprendizagem dos professores

\begin{tabular}{|l|l|}
\hline \multicolumn{1}{|c|}{ Desenvolvimento das aulas } & \multicolumn{1}{|c|}{ n (\%) } \\
\hline Poucas aulas desenvolvidas & $49(40,5 \%)$ \\
\hline Em parte, foram desenvolvidas as aulas & $29(24,0 \%)$ \\
\hline A maioria das aulas foi desenvolvida & $43(35,5 \%)$ \\
\hline \multicolumn{1}{|c|}{ Recursos utilizados durante o ensino remoto } & \\
\hline Material impresso & $102(84,3 \%)$ \\
\hline WhatsApp & $49(40,5 \%)$ \\
\hline Ambiente virtual de aprendizagem & $28(23,1 \%)$ \\
\hline Redes sociais & $14(11,6 \%)$ \\
\hline Ferramentas digitais & $14(11,6 \%)$ \\
\hline Videoconferência & $03(02,5 \%)$ \\
\hline Rádio & $01(0,8 \%)$ \\
\hline \multicolumn{1}{|c|}{ Percepção dos professores sobre a aprendizagem dos estudantes } \\
\hline Não conseguiram aprender ou aprenderam pouco & $84(69,4 \%)$ \\
\hline Conseguiram aprender satisfatoriamente & $30(24,8 \%)$ \\
\hline Conseguiram aprender bem & $07(05,8 \%)$ \\
\hline
\end{tabular}

Fonte: autores

Os dados revelaram que, durante o isolamento social, poucos foram os professores $(35,5 \%)$ que conseguiram ministrar suas aulas remotamente como o previsto. Esse dado demonstra os possíveis prejuízos para o processo ensino-aprendizagem dos estudantes no período do ensino remoto causado pelo isolamento social. Segundo Honorato e Marcelino (2020), as mudanças na prática docente impactaram a maneira de aprender do estudante, de modo que, no ensino remoto, a interação acerca de dúvidas e o retorno das atividades ainda são lacunas para muitos professores; por conseguinte, isso dificultou a avaliação em relação ao aprendizado do estudante e se este respondeu satisfatoriamente aos objetivos propostos pelas aulas.

Referente aos recursos utilizados para o processo ensino-aprendizagem, detectou-se a prevalência da utilização do material impresso (84,3\%) como principal ferramenta apontada pelos professores, seguido do WhatsApp (40,5\%). Esses achados podem sinalizar que a própria escola não estava preparada para o 
ensino remoto, como mencionado por Palú, Schütz e Mayer (2020), que também encontraram como principal material utilizado para as aulas remotas o impresso, devido à dificuldade de acesso dos professores às tecnologias e em razão das escassas ferramentas disponibilizadas pelos órgãos competentes, dificultando, assim, a execução das atividades de ensino-aprendizagem durante o ensino remoto e colaborando para a precarização das aulas da educação básica pública.

Quando verificada a percepção dos professores sobre a aprendizagem dos estudantes durante o ensino remoto, apenas 5,8\% relataram que eles aprenderam bem. Esse dado pode estar relacionado ao número de aulas desenvolvidas, bem como à carência de interação professor/estudante e de ferramentas pedagógicas para o desenvolvimento das aulas remotas, dificultando a aprendizagem dos estudantes. O estudo de Honorato e Marcelino (2020) aponta que a baixa aprendizagem dos estudantes, durante o ensino remoto, está intimamente ligada à dificuldade de acesso às tecnologias nas escolas públicas, que a exclusão digital atinge estudantes de baixa renda e condição econômica social precária, dessa forma, dificultando uma educação de qualidade. Para os autores, as oportunidades de acesso às tecnologias devem ser igualitárias, e a pandemia acabou evidenciando as desigualdades sociais entre escolas públicas e privadas (HONORATO; MARCELINO, 2020).

Quanto ao nível de competência digital dos professores, os dados revelaram a predominância de B1-Integradores, indicando que eles experimentam tecnologias digitais em diferentes contextos e utilizam-nas em suas aulas, conforme demonstra o Gráfico, que apresenta o perfil dos professores por nível de competência.

Gráfico: Níveis de competências digitais dos professores

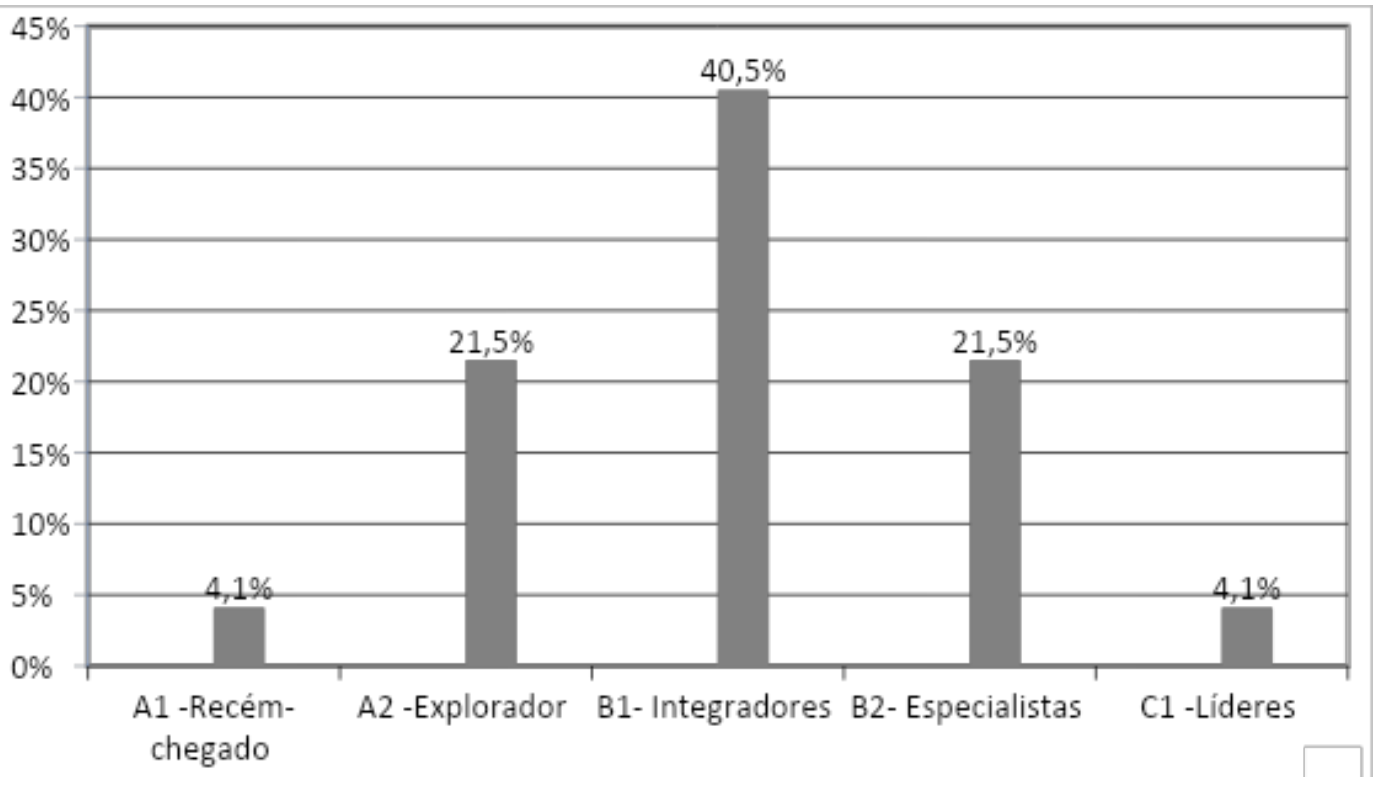

Segundo Dias-Trindade, Moreira e Nunes (2019), os professores pertencentes ao nível B1- integradores - utilizam criativamente as tecnologias para melhorar diversos aspectos do seu envolvimento profissional e estão dispostos a expandir seu repertório de prática. No entanto, ainda estão aprimorando a compreensão sobre que ferramentas funcionam melhor em que situações e sobre a adequação de tecnologias digitais a métodos e estratégias pedagógicas. Conforme os autores, esses professores precisam de mais algum tempo para experimentar e refletir, complementado por incentivo colaborativo e troca de conhecimento, para se tornarem especialistas.

O desenvolvimento das competências digitais nos professores é necessário para que possam preparar o estudante para o mundo digital. Conforme Le Boterf (2003) e Dias e Pinto (2020), a competência do professor é construída pela pessoa, a partir de um conjunto de recursos possíveis, e é preciso saber agir no 
desenvolvimento da competência. Segundo o autor Pinto (2020), o professor necessita de vários recursos para serem utilizados no processo de desenvolvimento de competência digital de docentes; é necessário ter conhecimentos, atitudes, habilidades, sendo um conjunto de vertentes inerentes à profissão, relacionadas com as aprendizagens e o ensino.

Além da proficiência digital geral, o DigCompEdu ainda proporciona verificar em qual área o professor possui maior ou menor competência, sendo estas divididas em seis: envolvimento profissional, recursos digitais, ensino e aprendizagem, avaliação, capacitação dos estudantes e promoção da competência digital dos estudantes. Para tanto, a Tabela 3, a seguir, apresenta os valores médios de cada área, sendo que estas variaram de zero a quatro.

\begin{tabular}{|l|l|}
\hline \multicolumn{2}{|c|}{ Tabela 3. Competência digital dos professores por área } \\
\hline Áreas de competências & Valores Médios \\
\hline Envolvimento Profissional & 1,9 pontos \\
\hline Capacitação dos estudantes & 1,9 pontos \\
\hline Avaliação & 1,8 pontos \\
\hline Tecnologia e Recursos Digitais & 1,7 pontos \\
\hline Ensino e Aprendizagem & 1,7 pontos \\
\hline Promoção dos estudantes & 1,5 pontos \\
\hline
\end{tabular}

Observa-se, na Tabela 3, maior média nas áreas de envolvimento profissional e capacitação dos estudantes (1,9 pontos), especificamente, a área de promoção dos estudantes (1,5 pontos). Estes dados indicam que os professores possuem mais proficiência digital para utilização profissional, como melhorar a comunicação entre os pares, potencializar estratégias na aprendizagem e partilhar e trocar materiais com colegas e estudantes, bem como utilizar as TDIC na educação com o objetivo de incorporar, potencializar e apoiar estratégias pedagógicas centradas no estudante e impulsionar o envolvimento ativo destes no processo de aprendizagem e sua apropriação dos conhecimentos. Por sua vez, possuem menos proficiência para promover a competência digital dos estudantes (DIAS-TRINDADE; MOREIRA; NUNES, 2019).

Acredita-se que o próprio momento pandêmico possa ter contribuído para o aperfeiçoamento das competências digitais dos professores, visto que foi necessário utilizar as TDIC para confeccionar o material didático, dessa forma, aproximando-os do uso diário das tecnologias. Em estudos semelhantes propostos por Martins (2020) e Oliveira, Gomes e Barcellos (2020), durante o ensino remoto, o envolvimento profissional do docente aumentou sua competência digital, pois Ihe proporcionou uma imersão diária nas tecnologias para garantir a continuidade do ensino através das aulas remotas. Os professores tiveram de se reinventar, diversificar e aprender novas experiências, aceitar as tecnologias como ferramenta de ensino, confeccionar seu material educativo, para tanto, permanecendo horas em frente ao computador (MARTINS, 2020).

Não obstante, Arruda (2020) e Martins (2020) reforçam que, mesmo em casa, com todas as angústias e dúvidas frente à necessidade de desenvolvimento das aulas e perante limitações tecnológicas, o anseio pela realização com qualidade contribuiu para a competência digital do professor.

\section{Conclusões}

Mediante os achados do presente estudo, pode-se inferir que os professores participantes se encontravam, em sua maioria, na competência digital B-1 Integrador; com isso, revelou-se que os docentes as utilizam de forma criativa e, ainda, melhoram a compreensão sobre as ferramentas e sobre a adequação de tecnologias digitais no que concerne a métodos e a estratégias pedagógicas. Ainda, entre as áreas de 
competência digital, os professores apresentaram maiores médias no envolvimento profissional e na capacitação dos estudantes.

No que tange ao desenvolvimento das aulas, poucos foram os professores que conseguiram ministrá-las remotamente como o previsto. E o recurso mais utilizado no processo de ensino-aprendizagem foi o material impresso, sendo seu compartilhamento mediado pelo WhatsApp.

Acredita-se que o próprio contexto do isolamento social tenha contribuído para aquisição de novas habilidades e competências frente às tecnologias por parte dos professores, visto que eles tiveram que ressignificar o ensino para a construção do conhecimento e da aprendizagem.

\section{Referências}

ARRUDA, E. P. Educação remota emergencial: elementos para políticas públicas na educação brasileira em tempos de Covid-19. Em Rede - Revista de Educação a Distância, v. 7, n. 1, p. 257-275, 2020. Disponível em: https://bit.ly/3nyHCNW. Acesso em: 15 mai. 2020.

AUTRAN, M. M. M.; BORGES, M. M. Competências Digitais: comportamentos, percepções e atitudes dos docentes/pesquisadores dos ppgcis - 2008 a 2012. In: XVII Encontro Nacional de Pesquisa em Ciência da Informação. 2016. Disponível em: https://bit.ly/32U6G9B. Acesso em: 19 jul. 2020.

BARDIN, L. Análise de conteúdo. Lisboa: Edições 70, 2011.

BRASIL. Base Nacional Comum Curricular: Educação Infantil e Ensino Fundamental. Brasília: MEC/Secretaria de Educação Básica, 2017.

BRASIL. Ministério da Saúde. Secretaria de Políticas de Saúde. Localiza SUS - Corona Vírus (COVID-19). Brasília, DF, 2020. Disponível em: https://bit.ly/3lBgXQg. Acesso em: 31 ago. 2020.

CASTAÑEDA, L.; ESTEVE, F.; ADELL, J. ¿Por qué es necesario repensar la competencia docente para el mundo digital?. Revista de Educación a Distancia, n. 56, p. 1-20, 2018. Disponível em: http://dx.doi. org/10.6018/red/56/6. Acesso em: 14 ago. 2020.

CHOI, M.; CRISTOL, D.; GIMBERT, B. Teachers as digital citizens: The influence of individual backgrounds, internet use and psychological characteristics on teachers' levels of digital citizenship. Computers \& Education, v. 121, n. 4, p. 143-161, 2018. Disponível em: https://doi.org/10.1016/j.compedu.2018.03.005. Acesso em: 05 jul. 2020.

DIAS, E.; PINTO, F. C. F. Educação e a Covid-19. Ensaio, Rio Janeiro, v. 28, n. 108, p. 545-554, 2020. Disponível em: https://bit.ly/32Sqcnl. Acesso em: 13 nov. 2020.

DIAS-TRINDADE, S.; MOREIRA, J. A.; NUNES, C. S. Escala de autoavaliação de competências digitais de professores. Procedimentos de construção e validação. Texto Livre: Linguagem e Tecnologia, Belo Horizonte, v.2, p. 72-91, mai/ago, 2019. Disponível em: https://bit.ly/3hJM4qG. Acesso em: 19 jul. 2020.

FERRARI, A. Digital competence in practice na analysis of Frameworks. Sevilla: JRC Spain: Institute for Prospective Technological Studies. European Commission. IPTS, 2012. Disponível em: https://bit. ly/3b884ZO. Acesso em: 19 ago. 2020.

GIL, A. C. Como elaborar projetos de pesquisa. 4a edição. São Paulo: Atlas S/A. 2012.

GOMES, V. T. S. et al. A Pandemia da Covid-19: Repercussões do Ensino Remoto na Formação Médica. Revista Brasileira de Educação Médica, Brasília, v. 44, n. 4, 2020. Disponível em: https://bit.ly/2UGADWR. Acesso em: 16 nov. 2020. 
HONORATO H. G; MARCELINO B. K. C. A. A arte de ensinar e a Pandemia Covid-19: a visão dos professores. REDE - Revista Diálogos em Educação, v. 1, n. 1, p. 208-220, 2020.

HONORATO, H. G. (no prelo). A educação brasileira e a pandemia covid-19: alinhavos entre o ensino remoto e a aprendizagem dos estudantes. 2020a.

LE BOTERF, G. Desenvolvendo a competência dos profissionais. São Paulo: Artmed, 2003.

MARTINS, R. X. A covid-19 e o fim da educação a distância: um ensaio. Em Rede - Revista de Educação a Distância, v. 7, n. 1, p. 242-256, 2020. Disponível em: https://bit.ly/3ICO9X6. Acesso em: 30 jul. 2020

OLIVEIRA, J. B. A.; GOMES, M.; BARCELLOS, T. A Covid-19 e a volta às aulas: ouvindo as evidências. Ensaio, Rio de Janeiro, v. 28, n. 108, p. 555-578, 2020. Disponível em: https://bit.ly/2ILVOzh. Acesso em: 13 nov. 2020.

PALÚ, J.; SCHÜTZ, A. J.; MAYER L. Desafios da educação em tempos de pandemia / organizadores: Janete Palú, Jenerton Arlan Schütz, Leandro Mayer. Cruz Alta: Ilustração, 2020.

REDECKER, C.; PUNIE, Y. European Framework for the Digital Competence of Educators (DigCompEdu). Luxembourg: Publications Office of the European Union, 2017. Disponível em: https://bit.ly/3gKh3BB. Acesso em: 30 jul. 2010. 